\title{
Role of Diet in Modification of Gut Microbiota in Obesity: Review of Recent Literature
}

\author{
Umama Khan ${ }^{1}$ S M Niazur Rahman² Nazmun Nahar Alam ${ }^{3 *}$
}

\author{
${ }^{1}$ Research Assistant, Center for Biomedical Research and Training (CBRT), Dhaka, Bangladesh \\ Email: umama@cbrtrain.org \\ ${ }^{2}$ Executive Director, Center for Biomedical Research and Training (CBRT), Dhaka, Bangladesh \\ Email: niazur@cbrtrain.org, ORCID id: 0000-0002-0584-0792 \\ ${ }^{3}$ Lecturer, Faculty of Medicine, AIMST University, Kedah, Malaysia \\ Email: nazmun1749@ gmail.com, ORCID id: 0000-0003-2408-7190 \\ Corresponding Author: \\ Dr. Nazmun Nahar Alam \\ Faculty of Medicine, AIMST University \\ 08100 Bedong, Kedah, Malaysia \\ Email: nazmun1749@gmail.com, Tel: +60163648502
}

\begin{abstract}
With the ever-increasing rate, obesity has become an epidemiological problem throughout the globe comprising about 39\% of the world population as of now. Among several reasons, disruption of the gut microbial ecosystem might contribute to the pathogenesis of metabolic disorders, including obesity, metabolic syndrome, type 2 diabetes, and other associated comorbidities. Though the mechanisms related to dysbiosis are unclear, diet might play a modulating role where different dietary approaches manipulate microbial richness and abundance as well as stability. For instance, shifting of Firmicutes and Bacteroidetes ratio in the gut might have a role in association with the dietary approaches and ingestion duration. Along with altered gut microbial composition, microbial metabolites such as short-chain fatty acids (SCFA) after ingestion of non-digestible dietary starches may have an impact on host metabolism by regulating lipogenesis, gluconeogenesis, and inflammation with potential associations to health and obesity. The dietary approaches like carbohydrates, fibre, protein, and/or fat diet at various arrangements can make a shift in the composition of gut microbiota if introduced for a short period. However, the unique pattern of the gut microbes usually remains the same along with the longer period of habitual diet. Though the short-term dietary intervention or circadian rhythm influences a transient change in gut microbes, other than habitual diet, the understanding related to long-term dietary changeinduced permanent alterations is minimum. Alternatively, the usage of prebiotics, probiotics as well as postbiotics could be beneficial to overcome dysbiosis. This review highlights the current knowledge and the interaction between the human intestinal microbiota and diet as a modifying factor, in obesity allowing the scientists to uncover novel targets and tools to use as customized therapy.
\end{abstract}


Key words: Diet, Obesity, Gut microbes, High-fiber diet, High fat diet INTRODUCTION

Without a doubt, obesity resulting from "positive energy balance" is becoming an epidemic posing a threat to public health throughout the world (1). Globally, the prevalence of obese and overweight individuals raised from a total of 857 million to 2.5 billion adults between 1980 to 2015 , respectively, which is about $39 \%$ of the world's total population as of now $(1,2)$. The other reasons comprise but are not limited to; sedentary lifestyle, epigenetics, pharmaceutics, different social and environmental variables, and gut microbes $(3,4)$. However, gut microbes not only play role in causing obesity but also have an impact on reducing the same $(4,5)$.

Gut microbiota is the collection of microorganisms residing in the intestinal tract composing an intricate host-gut relationship. Roughly, the human gut is the home of 10-100 trillion microbial cells, comprising immense diversity where most gut microbes identified using sequencing techniques though never been isolated through culture $(1,4)$. This diverse gut microbiota regulates a number of physiological phenomena in the host such as metabolism, immunology, vitamin and bile acid production, host genetic expression regulation, and many others $(4,6)$. For which, the alteration of these intestinal microbes alters these physiological processes driving towards disorders such as autoimmune diseases, inflammatory bowel diseases, obesity, and so on $(1,7)$. The composition of gut microbes is highly individualistic, a unique characteristic, which might have started from birth. The delivery mode, lactation period, and most importantly weaning have a greater influence in shaping the neonatal gut microbes dominated by Bifidobacterium $(8,9)$. In addition environmental influences, medications, and dietary changes during the first 3 years of age period result in the influx of new species and proliferation of the beneficial commensals (3). During that age period with the exploration of new food Bacteroidetes-related species amplifies whereas Bifidobacterium and Lactobacillus species and Enterobacteriaceae reduce (9).

After forming an adult-like microbial signature, it's essential to maintain homeostasis as disruption might be interconnected with obesity or other diseases, and diet can play a crucial role here $(3,8)$. One example of such interconnection might be the production of short-chain fatty acid (SCFA) by gut microbes after ingestion of non-digestible dietary starches. On the one hand, SCFA has an obesogenic role by providing extra energy through dietary caloric intake and also by depositing extra fat to the body (10). On the other hand, SCFA help to prevent obesity by modulating satiety response and lipid metabolism (4). Now this dual metabolic effect of SCFA might have a varied 
outcome in obese individuals as per ingestion duration and microbial alteration. Short-termed dietary intervention may rapidly alter the existing intestinal microbes in humans, which is usually a transient change $(3,7)$. Whereas long-term changes in the dietary pattern through the habitual diet may have an impact on a balanced gut microbial environment with the diversity and richness of the commensals, however, the study data are scarce (3).

As mentioned before, dysbiosis might be a causal factor for obesity, which was supported in an experiment where after feeding the same polysaccharide-rich diet there is a change of Bacteroidetes and Firmicutes ratio in the colon of ob/ob mice than its lean counterpart (11). Keeping in mind that factors related to dietary intervention such as selecting the type of diet, ingestion duration, and pattern, metabolites involved all these scientific data are paving a way to treat obesity through gut microbes, we have designed this review to understand the role of diet in modifying gut bacteria in obesity.

\section{OBESITY AND GUT MICROBIAL STATUS}

Primarily, the major phyla inhabiting the human intestinal tract are Firmicutes and Bacteroidetes, which is around $92.6 \%$ in the distal colon of C57BL/6J wild type mice (11). Other non-abundant phyla in the human gut include Actinobacteria (Bifidobacterium spp.), Proteobacteria (Escherichia coli, Helicobacter pylori), Fusobacteria, and also Verrucomicrobia (Akkermansia spp.) (4).

A shift of Firmicutes and Bacteroidetes ratio has been observed in ob/ob mice gut which includes $50 \%$ fewer Bacteroidetes than Firmicutes (11). This data was supported in studies both with animal and human models with decreasing Bacteroidetes and raising Firmicutes percentage (12, 13). However, an alternative scenario exists where diet act as the modulator in the Firmicutes-toBacteroidetes ratio. For instance, abundant Prevotella and Bacteroidetes (Xylanibacter) have been found while ingesting a high-fat western diet containing animal fat and sugar in comparison with a high-fiber diet with a significant amount of vegetable consumption where gut microbes revealed enriched Bacteroidetes and decreased Firmicutes (14). Again, in another diet-based experiment related to obesity, only the wild-type mice developed obesity where both germ-free mice and wildtype mice were fed the same high-fat diet. However, obesity was generated in germ-free mice when the obese microbiota was transplanted into them $(4,15)$.

\section{DIET - A MODULATING FACTOR IN OBESITY}




\section{High-Fibre Diet-Induced Changes and Metabolite Production}

To maintain a healthy body weight gut microbes yield energy from the host diet rich in dietary fibres such as vegetables, fruits, legumes, grains, nuts, and other plant-based edibles (3). Gut microbes such as Firmicutes produce metabolites like SCFA, acetate, propionate, and butyrate from these non-digestible dietary starches, the metabolic role of which usually benefit the health and consequently failing to regulate the energy expenditure could develop obesity $(4,10)$.

Non-obese individual harbour less SCFAs in feces than obese counterpart and is accompanied with a higher number of Firmicutes and fewer Bacteroidetes within the colon (13) (Table 1). One of the mechanisms associated might be the availability of increased colonic energy by SCFA promoting obesity along with the higher Firmicutes-Bacteroidetes ratio in the gut (10) (Table 1). Another mechanism of obesity through gut microbiota involves ligands for G-protein-coupled receptor GPR41 and GPR43 expressed in adipocytes, enteroendocrine cells, and mast cells where SCFA acts as a ligand for adipogenesis $(4,16)$.

\section{High-Fat Diet-Induced Dysbiosis and Endotoxemia}

Among different types of diets, those high in saturated fat such as butter, cheese, cream, lard, and oil are regarded as the primary cause of obesity. In male C57BL/6J mice fed with $72 \%$ high-fat diet reduces Bifidobacterium spp., Lactobacillus spp., and Prevotella spp.; and is related to the higher intestinal permeability and increased inflammatory biomarkers such as interleukin-1, tumor necrosing factor- $\alpha$ (17) (Table 1). While fed with 60\% fat, which includes $95 \%$ lard and $5 \%$ soybean oil, Sulfidogenic bacteria (Desulfobulbus species, Desulfobacter species; and Bilophila wadsworthia) raises in the gut of male C57BL/6J mice (18) (Table 1). Such dysbiosis is related to the over activation of the endocannabinoid system leading to increased gut permeability with a reduced number of zonulin and occludin, which are tight junction proteins in the intestine (4). This dysfunction in gut permeability triggers bacterial translocation into the bloodstream leading to endotoxemia with increased plasma lipopolysaccharide (LPS) and further contributing metabolic disturbances related to obesity (4) (Figure 1).

\section{Role of High-Protein Diet}

Usually, a high-protein diet with or without combination with a carbohydrate-restricted diet, causes loss of weight preventing obesity inducing an alteration of the gut microbes with a 
decreased abundance of Rosburia/Eubacterium rectale spp. (19) (Table 1). Again, excessive intake of dietary protein during childhood increases the "protein load" which may lead to obesity-like phenomenon in later life by changing insulin-like growth factor-1 (IGF-1) concentrations (20). Nonetheless, the current data related to a high-protein diet is yet not conclusive enough to elucidate the relationship between diet and obesity, for which it requires further investigation.

\section{Food Additives and Artificial Sweetening Agents}

Nowadays people are consuming processed food containing a high amount of artificial sweetening agents and food additives. Such type food additives may disrupt gut homeostasis by altering the gut microbiome or the mucus layer of the colon leading to obesity and other diseases (21). For instance, emulsifier carboxymethyl cellulose and polysorbate-80 directly affect Lactobacillus intestinalis, Lactobacillus murinus, Clostridium spp. and also Firmicutes bacterium composition in the gut (22) (Table 1). Moreover, higher ingestion of an artificial sweetener, acesulfamepotassium results in a significant rise of body weight in male CD-1 mice model by altering the gut microbiota and also by activating bacterial energy-yielding pathways (23) (Table 1). 
Table 1. Effects of different types of diet on gut microbes and their outcome on host health

\begin{tabular}{|c|c|c|c|c|}
\hline Reference & Study model & $\begin{array}{l}\text { Diet type and/or dietary } \\
\text { composition }\end{array}$ & $\begin{array}{l}\text { Changes in gut } \\
\text { microbiota }\end{array}$ & Effect on host health \\
\hline $\begin{array}{l}(13) \\
\text { Riva et. al., } \\
2017\end{array}$ & $\begin{array}{l}\text { Children with } \\
\text { obesity }(n=42)\end{array}$ & $\begin{array}{l}\text { Carbohydrates } 301.06 \mathrm{~g} / \text { day } \\
\text { Sugars } 110.16 \text { g/day }\end{array}$ & $\begin{array}{l}\uparrow \text { Firmicutes } \\
\downarrow \text { Bacteroidetes }\end{array}$ & 个SCFA \\
\hline $\begin{array}{l}(10) \\
\text { Rahat- } \\
\text { Rozenbloom } \\
\text { et al., } 2014\end{array}$ & $\begin{array}{l}\text { Human subject } \\
(\mathrm{n}=22)\end{array}$ & $\begin{array}{l}\text { Carbohydrates: } 244 \text { g/day } \\
\text { (lean), } 238 \text { g/day (obese). } \\
\text { Fiber: } 23.8 \text { g/day (lean), } 21.7 \\
\text { g/day (obese). } \\
\text { Protein: } 76 \text { g/day (lean), } 95 \\
\text { g/day (obese). } \\
\text { Fat: } 244 \text { g/day (lean), } 238 \\
\text { g/day (obese). }\end{array}$ & $\uparrow$ Firmicutes & $\uparrow$ SCFA \\
\hline $\begin{array}{l}(17) \\
\text { Cani et al., } \\
2008\end{array}$ & $\begin{array}{l}\text { C57BL/6J male } \\
\text { mice }\end{array}$ & $\begin{array}{l}72 \% \text { high-fat diet, } \\
28 \% \text { protein }\end{array}$ & $\begin{array}{l}\downarrow \text { Lactobacillus, } \\
\downarrow \text { Bifidobacterium, } \\
\downarrow \text { Prevotella }\end{array}$ & $\begin{array}{l}\text { } \uparrow \text { Intestinal permeability, } \\
\uparrow \text { Plasma LPS, } \\
\uparrow \text { Inflammatory } \\
\text { biomarkers (IL-1, TNF- } \alpha \text { ) }\end{array}$ \\
\hline $\begin{array}{l}(18) \\
\text { Shen et al., } \\
2014\end{array}$ & $\begin{array}{l}\text { C57BL/6J male } \\
\text { mice }\end{array}$ & $\begin{array}{l}60 \% \text { fat containing } 95 \% \text { lard } \\
\text { and } 5 \% \text { soybean oil }\end{array}$ & $\begin{array}{l}\uparrow \text { Desulfobulbus spp., } \\
\uparrow \text { Desulfobacter } \text { spp. } \\
\uparrow \text { Bilophila wadsworthia }\end{array}$ & $\begin{array}{l}\text { Impairment of tight } \\
\text { junction and macrophage } \\
\text { infiltration }\end{array}$ \\
\hline $\begin{array}{l}(19) \\
\text { Russell et al., } \\
2011\end{array}$ & $\begin{array}{l}\text { Humans with } \\
\text { obesity ( } n=17)\end{array}$ & $\begin{array}{l}\text { Two diet groups: } \\
\text { (1) High-protein and } \\
\text { moderate-carbohydrate diet: } \\
\text { Protein } 139 \mathrm{~g} / \text { day, fat } 82 \mathrm{~g} / \text { day, } \\
\text { and carbohydrate } 181 \mathrm{~g} / \mathrm{day} \text { ( } 4 \\
\text { weeks); } \\
\text { (2) High-protein and low } \\
\text { carbohydrate diet: } \\
\text { Protein } 137 \mathrm{~g} / \text { day, fat } 143 \\
\text { g/day, and carbohydrate } 22 \\
\text { g/day (4 weeks). } \\
\text { Before intervention: weight } \\
\text { maintenance diet: } \\
\text { Protein } 85 \mathrm{~g} / \text { day, fat } 116 \mathrm{~g} / \text { day, } \\
\text { and carbohydrate } 360 \mathrm{~g} / \mathrm{day}(7 \\
\text { days) }\end{array}$ & $\begin{array}{l}\downarrow \text { Roseburial } \\
\text { Eubacterium rectale }\end{array}$ & $\begin{array}{l}\downarrow \text { SCFA } \\
\downarrow \text { Ferulate, a fibre-derived, } \\
\text { antioxidant phenolic } \\
\text { acids }\end{array}$ \\
\hline $\begin{array}{l}\text { (22) } \\
\text { Chassaing et } \\
\text { al., } 2017\end{array}$ & $\begin{array}{l}\text { Mucosal simulator } \\
\text { of human intestinal } \\
\text { microbial ecosystem } \\
\text { (M-SHIME) model, } \\
\text { C57BL/6 mice }\end{array}$ & $\begin{array}{l}\text { Synthetic dietary emulsifiers } \\
\text { polysorbate } 80 \text { (P80) and } \\
\text { carboxymethylcellulose (CMC) }\end{array}$ & $\begin{array}{l}\uparrow \text { Proteobacteria } \\
\uparrow \text { Enterobacteriacae, } \\
\downarrow \text { Bacteroidaceae }\end{array}$ & $\begin{array}{l}\uparrow \text { Pro-inflammatory gene } \\
\text { expression } \\
\uparrow \text { Production of cytokines }\end{array}$ \\
\hline $\begin{array}{l}(23) \\
\text { Bian et al., } \\
2017\end{array}$ & CD-1 mice & $\begin{array}{l}\text { Acesulfame-potassium (Ace- } \\
\mathrm{K} \text { ), an artificial sweetener }\end{array}$ & $\begin{array}{l}\uparrow \text { Bacteroides, } \\
\uparrow \text { Anaerostipes, } \\
\uparrow \text { Sutterella }\end{array}$ & $\begin{array}{l}\text { } \uparrow \text { Weight gain, } \\
\uparrow \text { Sugar and xylose } \\
\text { transport, } \\
\uparrow \text { Glycolysis and TCA } \\
\text { cycle }\end{array}$ \\
\hline
\end{tabular}

(g: Gram, SCFA: Short chain fatty acid, LPS: Lipopolysaccharide, IL-1: Interleukin-1, TNF- $\alpha$ : Tumour necrosing factor- $\alpha$, TCA cycle: Tricarboxylic acid cycle, $\uparrow$ Increasing, $\downarrow$ Decreasing) 


\section{ROLE OF DIET TO MODULATE GUT MICROBIOTA CONCERNING THE TIME}

\section{Short-Term Dietary Intervention}

During short periods of dietary interventions, substantial changes in the microbiota have been observed. After consuming a plant-based diet rich in fibre from fruits and legumes, and an animalbased diet composing eggs, meats, and cheeses for about 24 hour period, rapid transient changes with increased levels of Bacteroides, Alistipes, and Bilophila, and decreased the levels of Firmicutes (Roseburia, Eubacterium rectale, and Ruminococcus bromii) was found in the human gut (24). However, altered gut microbes revert to their baseline state after the intervention period is over as the core microbial profile, especially the enterotypes are thought to remain stable in the intestine $(3,24)$. In another two-phased study in elderly obese women suggested transient microbial change where in the first phase pro-inflammatory components such as Collinsella raised and Lachnospiraceae and Ruminococcaceae which produces SCFA reduced. In the second phase, after intervening in the diet with nutritional supplementation for about 15 days, Collinsella decreased and Akkermansia increased significantly. Though this diversion was short-lived they are crucial to adjust severely disrupted intestinal microbes in elderly obese women usually related to metabolic health (25).

\section{Habitual Diet as a Long-Term Diet}

Unlike the short-term transient dietary intervention, long-term dietary patterns play a role to form an individual's core gut microbial profile which is usually a persistent one (25). Though there are several modulating factors like lifestyle, appetite, accessibility; habitual diet is regarded as the long-term diet, which shapes the gut microbes starting from the preadolescence period (3). Yet, it remains uncertain about how lengthy a dietary modification would require for the permanent change of the microbial ecology (3). People in western countries following high-fibre and highcarbohydrate diets have more Prevotella whereas people consuming high-fat and high-protein diets are enriched with Bacteroides $(3,26)$. Again, it is crucial to understand whether people having the Bacteroides enterotype have a higher incidence of diseases with specific types of diet. If an enterotype is found to be associated with any disease or disease-like condition such as obesity, then long-term dietary interventions may be modulated to enhance health (26). However, the current data related to a long-term dietary intervention is not convincing enough to interlink it with gut microbial perturbation and obesity requiring further exploration. 


\section{Circadian Rhythm Associated Alteration}

Though gut microbes are not exposed to the light and dark cycle, their functional characteristic and arrangement can still be affected by circadian rhythms through intrinsic circadian clocks (3, 27). The reasons associated might be the availability of nutrients, auto-antibodies, and peptides; their variation can relate to diurnal alteration of rhythmic histone acetylation by epithelial histone deacetylase 3 (HDAC3). This HDAC3 assimilates microbial and circadian signals which in turn affects nutrient intake further disrupting microbes leading towards diet-induced obesity (28).

Furthermore, interruption in sleep patterns found in jet lag and shift workers is another common reason for changing the body's internal clock and eating habits (3). About $15 \%$ of commensal bacterial taxa in the gut change throughout a day with periodic consumption of food leading towards microbial abundance (27). However, the same study suggests microbial dysbiosis in both mice and humans due to circadian misalignment promoting glucose intolerance and obesity (27)

\section{USAGE OF PREBIOTICS, PROBIOTICS, AND POSTBIOTICS TO MODULATE GUT MICROBES - TO MANAGE OBESITY}

Since gut microbiota is responsible not only for triggering obesity but also is a potential tool to treat it, so adding prebiotics and probiotics to the diet renders an option to modulate the growth of beneficial microbes to manage obesity (7).

Prebiotics are non-digestible dietary fibre such as inulin, galactooligosaccharides (GOS), betaglucans, and fructooligosaccharides (FOS) that are fermented by host gut bacteria after arriving at the colon (3). During this process, gut microbial composition changes, for example, adding a mixture of fructooligosaccharides and inulin $(10 \mathrm{~g} / \mathrm{d})$ to the diet increases the count of bifidobacteria, mostly Bifidobacterium adolescentis (29). Again, such fermentation process produces SCFAs such as acetate, propionate, and butyrate which can counteract the overexpression of several host targets causing obesity $(3,30)$.

Probiotics are live microorganisms, such as bacteria Lactobacillus and Bifidobacterium, Akkermansia. muciniphila or the yeast Saccharomyces boulardii, the composition of which may differentially affect the microbial arrangement or directly impact the host, by reinforcing gut barrier, reducing inflammation, improving glucose homeostasis eventually reduces obesity (31) (Figure 1). For instance, a 10-week dietary interference in mice models consuming a high-fat diet 
with the probiotic Lactobacillus paracasei ssp. paracasei F19 raises circulating angiopoietin-like protein 4 (Angptl4) levels and considerably decreases triglyceride deposition into adipocytes ultimately reducing obesity (32).

Another is postbiotics which include purified bacterial metabolites like SCFA or other bacterial constituents having a distinct advantage to the host. Although the active use of postbiotics is relatively a new approach, studies suggested that utilization of bacterial metabolites as postbiotics would remove the necessity to administer live bacteria comprising potentially unknown pathogens (7).

The summarized view of this review has presented schematically in Figure 1. It shows, gut microbial alteration due to dietary intervention leads to obesity. Both diet types and ingestion duration may cause changes in gut microbial composition. These alterations can lead to obesity by glucose intolerance, changing concentration of bacterial derived metabolites, changing gut permeability, causing endotoxemia, lipogenesis. And, introduction of prebiotics, probiotics, and postbiotics also alter the gut microbes, however these changes work to reinforce gut barrier, reduce inflammation and to improve glucose homeostasis eventually reducing obesity.

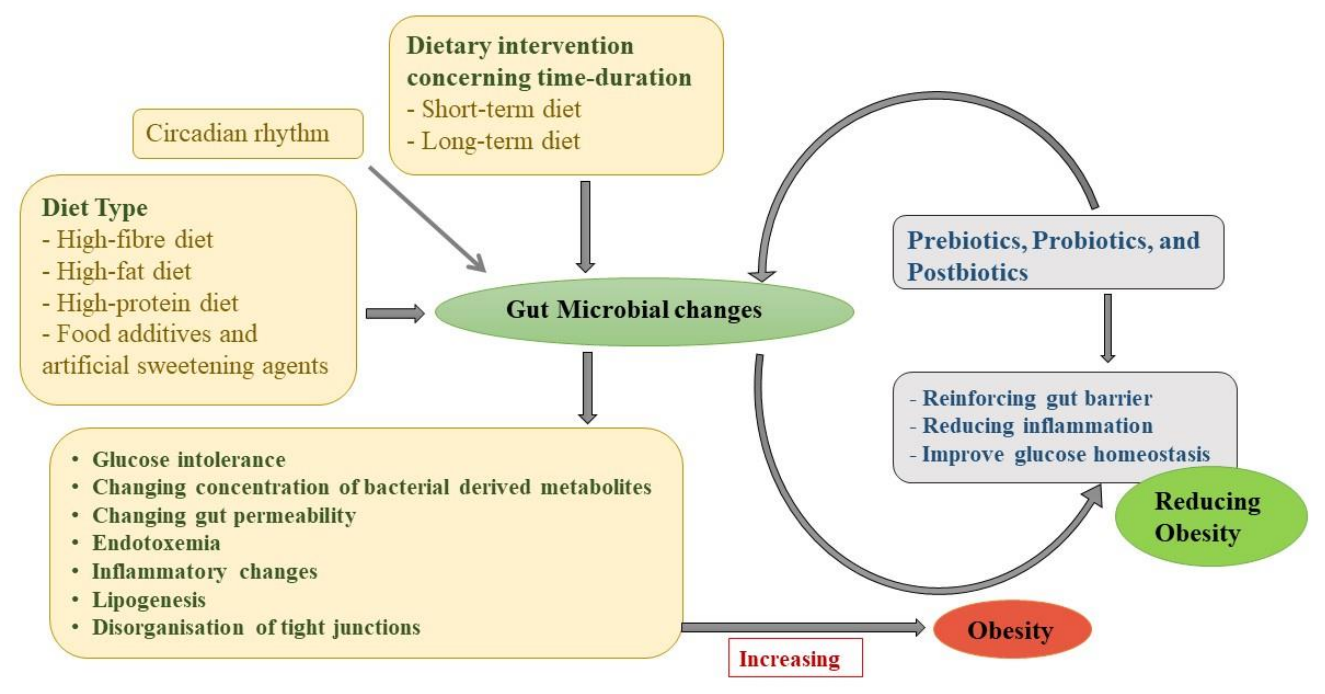

Figure 1. Gut microbial alteration due to dietary intervention leading to obesity. Diet types and ingestion duration may cause changes in gut microbial composition. These alterations can lead to obesity by glucose intolerance, changing concentration of bacterial derived metabolites, changing gut permeability, causing endotoxemia, lipogenesis. Again, introduction of prebiotics, probiotics, and postbiotics also alter the gut microbes, however these changes work to reinforce gut barrier, reduce inflammation and to improve glucose homeostasis eventually reducing obesity. 


\section{CONCLUSION}

Gut microbiota has close relations to develop obesity, as they alter rapidly to some extent responding to modification of diet and lifestyle. Additionally, the diet type and the duration of intervention are crucial factors to consider, as the response of the human gut microbiota to such changes is highly specific. To manage obesity and other accompanying comorbidities, maintenance of gut microbial homeostasis is essential by its modulation through diet, lifestyle modification, and by using prebiotics, probiotics, and postbiotics. Though there are some promising results related to this issue in animal models, study data are scarce in human subjects. For the proper understanding of the role of diet more studies are required to design and implement novel strategies to manage obesity by alteration of gut microbial composition.

\section{REFERENCES}

1. Vallianou N, Stratigou T, Christodoulatos GS, Dalamaga M. Understanding the Role of the Gut Microbiome and Microbial Metabolites in Obesity and Obesity-Associated Metabolic Disorders: Current Evidence and Perspectives. Current obesity reports. 2019;8(3):317-32.

2. Chooi YC, Ding C, Magkos F. The epidemiology of obesity. Metabolism. 2019;92:6-10.

3. Leeming ER, Johnson AJ, Spector TD, Le Roy CI. Effect of Diet on the Gut Microbiota: Rethinking Intervention Duration. Nutrients. 2019;11(12):2862.

4. Cuevas-Sierra A, Ramos-López O, Riezu-Boj J, Milagro F, Martínez J. Diet, Gut Microbiota, and Obesity: Links with Host Genetics and Epigenetics and Potential Applications. Advances in nutrition. 2019;10 suppl_1:S17-S30.

5. Genton L, Mareschal J, Charretier Y, Lazarevic V, Bindels LB, Schrenzel J. Targeting the Gut Microbiota to Treat Cachexia. Frontiers in cellular and infection microbiology. 2019;9:305.

6. Rowland I, Gibson G, Heinken A, Scott K, Swann J, Thiele I, et al. Gut microbiota functions: metabolism of nutrients and other food components. Eur J Nutr. 2018;57(1):1-24.

7. Ojeda P, Bobe A, Dolan K, Leone V, Martinez K. Nutritional modulation of gut microbiota - the impact on metabolic disease pathophysiology. J Nutr Biochem. 2016;28:191-200.

8. Tanaka M, Nakayama J. Development of the gut microbiota in infancy and its impact on health in later life. Allergology international : official journal of the Japanese Society of Allergology. 2017;66(4):515-22.

9. Voreades N, Kozil A, Weir TL. Diet and the development of the human intestinal microbiome. Front Microbiol. 2014;5:494.

10. Rahat-Rozenbloom S, Fernandes J, Gloor GB, Wolever TMS. Evidence for greater production of colonic short-chain fatty acids in overweight than lean humans. Int J Obes (Lond). 2014;38(12):1525-31.

11. Ley RE, Bäckhed F, Turnbaugh P, Lozupone CA, Knight RD, Gordon JI. Obesity alters gut microbial ecology. Proceedings of the National Academy of Sciences of the United States of America. 2005;102(31):11070-5.

12. Walters WA, Xu Z, Knight R. Meta-analyses of human gut microbes associated with obesity and IBD. FEBS Lett. 2014;588(22):4223-33. 
13. Riva A, Borgo F, Lassandro C, Verduci E, Morace G, Borghi E, et al. Pediatric obesity is associated with an altered gut microbiota and discordant shifts in Firmicutes populations. Environmental microbiology. 2017;19(1):95-105.

14. De Filippo C, Cavalieri D, Di Paola M, Ramazzotti M, Poullet JB, Massart S, et al. Impact of diet in shaping gut microbiota revealed by a comparative study in children from Europe and rural Africa. Proceedings of the National Academy of Sciences of the United States of America. 2010;107(33):14691-6.

15. Bäckhed F, Manchester JK, Semenkovich CF, Gordon JI. Mechanisms underlying the resistance to diet-induced obesity in germ-free mice. Proceedings of the National Academy of Sciences of the United States of America. 2007;104(3):979-84.

16. Karaki S, Tazoe H, Hayashi H, Kashiwabara H, Tooyama K, Suzuki Y, et al. Expression of the short-chain fatty acid receptor, GPR43, in the human colon. Journal of molecular histology. 2008;39(2):135-42.

17. Cani PD, Bibiloni R, Knauf C, Waget A, Neyrinck AM, Delzenne NM, et al. Changes in Gut Microbiota Control Metabolic Endotoxemia-Induced Inflammation in High-Fat Diet-Induced Obesity and Diabetes in Mice. Diabetes. 2008;57(6):1470.

18. Shen W, Wolf PG, Carbonero F, Zhong W, Reid T, Gaskins HR, et al. Intestinal and systemic inflammatory responses are positively associated with Sulfidogenic bacteria abundance in high-fat-fed male C57BL/6J mice. The Journal of nutrition. 2014;144(8):1181-7.

19. Russell WR, Gratz SW, Duncan SH, Holtrop G, Ince J, Scobbie L, et al. High-protein, reduced-carbohydrate weight-loss diets promote metabolite profiles likely to be detrimental to colonic health. Am J Clin Nutr. 2011;93(5):1062-72.

20. Xu S, Xue Y. Protein intake and obesity in young adolescents (Review). Exp Ther Med. 2016;11(5):1545-9.

21. Gerasimidis K, Bryden K, Chen X, Papachristou E, Verney A, Roig M, et al. The impact of food additives, artificial sweeteners and domestic hygiene products on the human gut microbiome and its fibre fermentation capacity. Eur J Nutr. 2020;59(7):3213-30.

22. Chassaing B, Van de Wiele T, De Bodt J, Marzorati M, Gewirtz AT. Dietary emulsifiers directly alter human microbiota composition and gene expression ex vivo potentiating intestinal inflammation. Gut. 2017;66(8):1414-27.

23. Bian X, Chi L, Gao B, Tu P, Ru H, Lu K. The artificial sweetener acesulfame potassium affects the gut microbiome and body weight gain in CD-1 mice. PLOS ONE. 2017;12(6):e0178426.

24. David LA, Maurice CF, Carmody RN, Gootenberg DB, Button JE, Wolfe BE, et al. Diet rapidly and reproducibly alters the human gut microbiome. Nature. 2014;505(7484):559-63.

25. Cancello R, Turroni S, Rampelli S, Cattaldo S, Candela M, Cattani L, et al. Effect of ShortTerm Dietary Intervention and Probiotic Mix Supplementation on the Gut Microbiota of Elderly Obese Women. Nutrients. 2019;11(12):3011.

26. Wu GD, Chen J, Hoffmann C, Bittinger K, Chen Y-Y, Keilbaugh SA, et al. Linking longterm dietary patterns with gut microbial enterotypes. Science (New York, NY). 2011;334(6052):105-8.

27. Thaiss CA, Zeevi D, Levy M, Zilberman-Schapira G, Suez J, Tengeler AC, et al. Transkingdom control of microbiota diurnal oscillations promotes metabolic homeostasis. Cell. 2014;159(3):514-29.

28. Kuang Z, Wang Y, Li Y, Ye C, Ruhn KA, Behrendt CL, et al. The intestinal microbiota programs diurnal rhythms in host metabolism through histone deacetylase 3. bioRxiv. 2019:580613. 
29. Barczynska R, Bandurska K, Slizewska K, Litwin M, Szalecki M, Libudzisz Z, et al. Intestinal Microbiota, Obesity and Prebiotics. Polish journal of microbiology. 2015;64(2):93-100. 30. Delzenne NM, Neyrinck AM, Bäckhed F, Cani PD. Targeting gut microbiota in obesity: effects of prebiotics and probiotics. Nature Reviews Endocrinology. 2011;7(11):639-46.

31. Wieërs G, Belkhir L, Enaud R, Leclercq S, Philippart de Foy JM, Dequenne I, et al. How Probiotics Affect the Microbiota. Frontiers in cellular and infection microbiology. 2019;9:454.

32. Aronsson L, Huang Y, Parini P, Korach-André M, Håkansson J, Gustafsson J-Å, et al. Decreased fat storage by Lactobacillus paracasei is associated with increased levels of angiopoietin-like 4 protein (ANGPTL4). PloS one. 2010;5(9):e13087. 